\title{
Repression of the miR-93-enhanced sensitivity of bladder carcinoma to chemotherapy involves the regulation of LASS2
}

This article was published in the following Dove Press journal:

OncoTargets and Therapy

29 March 2016

Number of times this article has been viewed

\author{
Jingyu Liu* \\ Haifeng Wang* \\ Yan Wang \\ Zhenkun Li \\ Yi Pan \\ Qiying Liu \\ Mingying Yang \\ Jiansong Wang
}

Department of Urology, The Second Affiliated Hospital of Kunming Medical University, Yunnan Institute of Urology, Yunnan Province, People's Republic of China

*These authors contributed equally to this work
Correspondence: Jiansong Wang

Department of Urology, The Second Affiliated Hospital of Kunming Medical University, Yunnan Institute of Urology, No 347 Dianmian Street, Kunming 65010I, Yunnan Province, People's Republic of China

$\mathrm{Tel}+86|80838| 0913$

Fax +86 87| 65I6655 I

Email jiansongwangkm@।26.com

\begin{abstract}
The aberrant expression of miRNA has an important function in bladder cancer (BC). Previous studies indicate that LASS2 is involved in the development of sensitivity to chemotherapy in cancer cells. In the present study, the miRNAs related to LASS2 were selected by using miRNA profiling to distinguish chemo-resistant and chemo-sensitive tumor specimens from patients. Higher levels of miR-93 were observed in the cisplatin-resistant BC cell line RT4, compared to the cell line T24. The role of miR-93 in chemo-sensitivity was demonstrated both in cell culture and mouse tumor xenograft models. We found that inhibiting miR-93 promoted cisplatin-induced apoptosis due to the accumulation of DNA damage. A reporter gene assay was performed, and the results showed miR-93 was not a target of the 3' untranslated region of LASS2, but had an altered protein expression level. Inhibitors of miR-93 could also enhance the chemo-sensitivity of tumor cells transfected with si-LASS2, but the effect was very slight. These findings suggest that miR-93 plays an important role in the chemo-sensitivity of BC, and may be involved in regulating the LASS2 gene.
\end{abstract}

Keywords: miRNA, inhibition, chemo-sensitivity, bladder cancer

\section{Introduction}

Bladder carcinoma $(\mathrm{BC})$ is the most common malignancy of the urinary tract. Although patients with $\mathrm{BC}$ generally have a good prognosis, it has the highest recurrence rate of any malignancy. ${ }^{1}$ At present, Bacillus Calmette-Guerin is still the first line therapy for both non-muscle-invasive bladder cancer and muscle-invasive bladder cancer. ${ }^{2}$ However, accumulating evidence suggests that transurethral resectioning of bladder tumors followed by platinum-based chemotherapy could be an alternative treatment for patients with muscle-invasive bladder cancer. ${ }^{3-5}$ The majority of patients with $\mathrm{BC}$ ultimately fail chemotherapy, and the main cause is chemotherapy resistance. ${ }^{6,7}$ Clinically, the identification and treatment of chemo-resistant $\mathrm{BC}$ remains an unsolved problem.

miRNAs are a class of small, non-coding RNAs that negatively regulate gene and protein expression at the post-transcriptional level. They bind with the $3^{\prime}$ untranslated regions (UTRs) in human mRNAs and induce mRNA degradation and inhibit translation. ${ }^{8-10}$ It is estimated that $30 \%$ of all genes are regulated by miRNAs. ${ }^{11}$ MiRNAs have been reported to be dysregulated in tumors and regulate potent oncogenes or tumor suppressor genes. ${ }^{12,13}$ The dysregulation of miRNAs has also been reported to be responsible for BC progression and metastasis. ${ }^{14,15}$ In addition, miRNAs could be a potential target for chemotherapy in BC. For example, miRNA-27a could modulate cisplatin resistance in bladder cancer by targeting SLC7A11. ${ }^{16}$ Increased miR-34a 
could cause chemo-sensitization. ${ }^{17}$ Nordentoft et al profiled the expression of 671 miRNAs from $\mathrm{BC}$, and found that a decrease in miR-138 and an increase in miR-27a and miR-642 generally increased sensitivity to cisplatin. ${ }^{18}$ In previous studies, we discovered that LASS2 can effectively inhibit the growth and metastasis of bladder cancer. Additionally, LASS2 was found to affect chemo-sensitization in several types of cancer, including breast cancer. ${ }^{19}$ However, little is known about the influence of LASS2-related miRNAs on chemotherapy of BC.

The purpose of this study was the investigation of the regulation of LASS2 expression by miR-93, which could cause chemo-sensitization in BC. Furthermore, the biological functions of dysregulated miR-93 and LASS2 in $\mathrm{BC}$ were also investigated. The results of this study could elucidate the mechanism of miR-93 and LASS2 in chemotherapy of BC.

\section{Materials and methods}

\section{Human tissue specimens, cell culture, and transfections}

Tumor specimens from ten patients with a histological diagnosis of BC at Kunming Medical University were utilized. The tumor samples were obtained from patients who had similar characteristics, and received the same standard chemotherapy. Chemo-sensitive and chemo-resistant cases were distinguished based on the histology of residual tumor cells in bladder biopsies taken after the completion of chemotherapy. All patients gave informed consent before the specimens were taken, and the study protocols were approved by the the Ethical Committee of Kunming Medical University, People's Republic of China.

\section{MiRNA microarrays}

Microarray experiments were performed at KangChen Bio-tech Inc at Shanghai. Briefly, total RNA was prepared from tissue using the mirVana kit from Ambion (Austin, TX, USA), and subsequently labeled and hybridized using Agilent's miRNA Complete Labeling and Hyb Kit (Agilent Technologies, Santa Clara, CA, USA). Arrays were scanned on an Agilent Microarray Scanner and the data were extracted using the Agilent Feature Extraction Software.

\section{Cell culture and treatment}

Human bladder cancer cell lines RT4 and T24 were cultured in Dulbecco's Modified Eagle's Medium/F12 medium supplemented with $10 \%$ fetal bovine serum and antibiotics at $37^{\circ} \mathrm{C}$ with $5 \% \mathrm{CO}_{2}$. The cells were subcultured every 3 days using a trypsin/ethylenediaminetetraacetic acid solution (saline containing $0.05 \%$ trypsin, $0.01 \mathrm{M}$ sodium phosphate, and $0.53 \mu \mathrm{M}$ ethylenediaminetetraacetic acid, $\mathrm{pH}$ 7.4). Cisplatin was dissolved in dimethyl sulfoxide $(10 \mathrm{mg} / \mathrm{mL})$ (Sigma-Aldrich Co., St Louis, MO, USA) and added to cells at a final concentration of $10 \mu \mathrm{M}$.

\section{Quantitative reverse transcription- polymerase chain reaction (RT-PCR)}

Total RNA was extracted using TRIzol reagent (Thermo Fisher Scientific, Waltham, MA, USA), and cDNAs were synthesized using the PrimeScript RT reagent Kit (TaKaRa, Dalian, People's Republic of China). RT-PCR analyses of mRNA were performed with SYBR Mix (TOYOBO, Shanghai, People's Republic of China). miRNA expression was quantified using the PrimeScript miRNA RT-PCR kit (TaKaRa), and was normalized with U6 small nuclear RNA. The concentration in each sample was calculated using the 2- $\Delta \Delta \mathrm{Ct}$ method. The primers were: miR-93, forward: CTAGCCTGCAGGGGTGAGTGGTGGGTCCCTGT; LASS2 forward: GCCTTGCTCTTCCTCATCGTTC, reverse: TGCTTGCCACTGGTCAGGTAGA.

\section{Western blot}

Cell lysates were resolved via sodium dodecyl sulfatepolyacrylamide gel electrophoresis, transferred to a nitrocellulose membrane, and blocked with phosphate-buffered saline $(\mathrm{PBS}) /$ Tween-20 containing 5\% non-fat milk. The membrane was incubated with antibodies for LASS2 (Santa Cruz Biotechnology Inc., Dallas, TX, USA) or $\beta$-actin (Santa Cruz Biotechnology Inc.). Protein signals were detected using enhanced chemiluminescence (Pierce, Rockford, IL, USA).

\section{MiRNA inhibitor/siRNA and transfection}

MiR-93 inhibitors and antagomirs were synthesized by Ribo (Guangzhou, People's Republic of China), then transfected into cells using Lipofectamine 2000 reagent (Thermo Fisher Scientific) according to the manufacturer's instructions. LASS2 shRNA plasmid was constructed and reserved by our laboratory.

\section{Clonogenic assay for cell survival}

The drug sensitivity of the cells was assessed by their ability to form colonies. In brief, approximately $1.0 \times 10^{4}$ cells were seeded into a $60 \mathrm{~mm}$ tissue culture dish and incubated overnight, after which the cells were treated with cisplatin $(0-10 \mu \mathrm{M})$ for 24 hours. The cells were washed in PBS and cultured for 7 days under a $5 \% \mathrm{CO}_{2}$ atmosphere in air at $37^{\circ} \mathrm{C}$. The colonies were fixed with $100 \%$ methanol for 10 minutes, and stained with Giemsa in phosphate buffer 
(pH 6.4). Colonies composed of 50 or more cells were scored as survivors, and the surviving fraction for a given treatment dose was calculated as the relative plating efficiency of treated versus untreated (control) cultures. All experiments were performed three times and yielded similar results.

\section{Apoptosis assays}

Apoptosis was determined by measuring the activation of CASP3 using the caspase assay kit (Promega Corporation, Fitchburg, WI, USA), and cleaved CASP3 protein was detected via Western blotting.

\section{DNA damage assays}

Cells were seeded at $2 \times 10^{4} /$ well in a black 96 -well plate with a clear flat bottom (Costar; Corning Incorporated, NY, USA). After treatment, the cells were harvested and fixed with $4 \%$ formaldehyde in PBS for 15 minutes at room temperature, and permeabilized. Nonspecific binding was blocked by incubating the cells with $1 \%$ BSA and $0.02 \%$ Triton X-100 in PBS for 20 minutes at room temperature. The cells were sequentially incubated with anti- $\gamma \mathrm{H} 2 \mathrm{AX}$ antibody (1:500; Santa Cruz Biotechnology Inc.) for 2 hours at room temperature, fluorescein isothiocyanate-conjugated anti-rabbit $\operatorname{IgG}$ antibody (1:500; Thermo Fisher Scientific) for 1.5 hours, and 4',6-diamidino2-phenylindole (10 $\mu \mathrm{g} / \mathrm{mL}$; Thermo Fisher Scientific) for 10 minutes. The cells were washed three times with $0.02 \%$ Triton X-100 in PBS for 10 minutes each time and visualized using ImageXpress (Molecular Devices LLC, Sunnyvale, CA, USA). The number and the total area of $\gamma \mathrm{H} 2 \mathrm{AX}$ foci in images were measured via ImageXpress. Images of stained cells were acquired from an automated fluorescence microscope platform in ImageXpress using a 40× objective lens.

\section{In vivo tumorigenesis assay}

Five-week-old BALB/c nude mice for the in vivo tumorigenesis assay were purchased from the Animal Center for Vitalriver (Beijing, People's Republic of China), and randomly assigned to one of four groups (ten mice per group). All animals were maintained in a 12:12 h light/dark cycle, housed in individual cages with fresh food and water ad libitum. All animal-handling procedures were performed according to the Guide for the Care and Use of Laboratory Animals of the National Institutes of Health and followed the guidelines of the Animal Welfare Act. The study was approved by the the Ethics Committee of Kunming Medical University. RT-4 cells $\left(5 \times 10^{6}\right.$ cells $)$ that were transiently transfected with miR-93 antagomir or miR-NC antagomir were suspended in $100 \mu \mathrm{L}$ Roswell Park Memorial Institute medium 1640 and injected subcutaneously in the right armpit of mice. After 10 days, tumor growth was monitored and when tumors reached $8 \mathrm{~mm}$ in diameter, the mice bearing miR-93-down-expressed tumors were randomly divided into two groups. One group received cisplatin treatment, and the other group served as a control. The same experiment was performed with miR-NC expressing tumors. Tumor sizes were recorded every 3 days. After 42 days, the mice were sacrificed for evaluation.

\section{Luciferase reporter assay}

A mixture of 100 ng pLUC-UTR, 50 ng-miR-93 mimics, and 10 ng Renilla was co-transfected into HEK293T cells; and firefly and Renilla luciferase activities were measured after 48 hours using a Dual-Luciferase Reporter Assay System (Promega Corporation) according to the manufacturer's protocol.

The Xho I and BamH I primers shown below were used to amplify the entire 3'UTR of LASS2.

LASS2-UTR-FTR: 5'-caaccetcgagACCCCATCCTCA ATAACA-3'

LASS2-UTR-RTR: 5'-aaggatccGCAAGGAAGGC ATAAGAAAGACTC-3'.

\section{Statistical analysis}

Each experiment was performed in triplicate. All data are presented as the mean \pm standard deviation. The differences between the means were analyzed with Student's $t$-test, a nonparametric Mann-Whitney $U$-test or an analysis of variance. All tests performed were two-sided. $P<0.05$ was considered to be statistically significant.

\section{Results}

\section{Expression of miRNAs probably targeting LASS2 in chemo-sensitivity}

To explore whether miRNAs had an effect on bladder cancer therapy, we first compared miRNA expression in five control tumor specimens with five chemo-resistant tumor specimens using microarrays. All of these specimens were from patients with stage II bladder cancer who received chemotherapy. The clinical and pathological characteristics of these ten patients are shown in Table S1, and the differentially-expressed miRNAs are shown in Table S2.

Computer algorithms were used to investigate the putative miRNAs that regulated the expression of LASS2. Three miRNAs, miR-93, miR-133a, and miR-101, were found to contain a nucleotide sequence complementary to the LASS2 3'UTR. The miRNA miR-101 has been clearly confirmed to affect the chemo-sensitivity of bladder cancer, while miR133a has been confirmed to inhibit proliferation. Thus, we 
excluded miR-133a and miR-101, then chose miR-93 for further investigation of its probable effects on chemo-sensitivity. The association of miR-93 with chemo-sensitivity in the two bladder cancer cell lines had different degrees of sensitivity to chemotherapy. As shown in Figure 1A, T24 cells were more sensitive to cisplatin treatment than RT4 cells, and had a lower expression of miR-93 than RT4 cells (Figure 1B). These results suggest a possible role for miR-93 in modulating the sensitivity of bladder cancer to chemotherapy.

\section{Suppression of miR-93 enhanced sensitivity of cancer cells to chemotherapy both in vitro and in vivo}

To determine the effect of miR-93 expression on the sensitivity of tumor cells to chemotherapy, we transfected RT4 and T24 cells with an miR-93 inhibitor or a control inhibitor. As shown in Figure 2A and B, treatment with the miR-93 inhibitor increased the sensitivity of the tumor cells to chemotherapy treatment. The enhanced effect of the repression of miR-93 on chemo-sensitivity was also observed in animal tumor models. As shown in Figure 2C and D, without cisplatin treatment, the tumors grew similarly in the presence or absence of miR-93 antagomir, and the tumors had approximately the same weight and volume. In contrast, following cisplatin treatment, the tumor weight and volume in the presence of miR-93 repression was significantly decreased as compared with that of the tumors in the absence of miR-93, suggesting that suppression of miR-93 enhanced the sensitivity of bladder cancer to chemotherapy.

\section{Repressing miR-93 promotes chemo- induced cell apoptosis via the accumulation of DNA damage}

We next explored the mechanism by which miR-93 modulated the sensitivity of the tumor cells to cisplatin treatment. We first determined whether or not the inhibitory effect of miR-93 on tumor sensitivity to cisplatin was associated with its effects on tumor cell proliferation. No difference in proliferation was found between the cells in which miR-93 was repressed and those in which it was not repressed (Figure 3A). However, an miR-93 inhibitor promoted the induction of apoptosis in tumor cells treated with cisplatin, as demonstrated by increased CASP3 activity in the cells transfected with the miR-93 inhibitor, as compared with cells transfected with a control inhibitor (Figure 3B). A Western blot analysis showed that 24 hours after treatment, CASP3 protein expression in RT4 and T24 cells transfected with miR-93 inhibitor was higher than that in cells transfected with the control inhibitor (Figure 3C). These results indicated that miR-93 may protect cells from chemotherapy-induced damage by inhibiting apoptosis. Next, we examined the role of DNA damage induction by the enhanced effect of miR-93 in chemo-sensitivity using immunofluorescence staining of $\gamma-\mathrm{H} 2 \mathrm{AX}$. No difference was found in the percentage of the cells with $\gamma-\mathrm{H} 2 \mathrm{AX}$ foci for both T24 and RT4 cells with or without miR-93 inhibitor, but after cisplatin treatment, the percentage of cells with $\gamma-\mathrm{H} 2 \mathrm{AX}$ foci increased for both T24 and RT4 cells transfected with miR-93 inhibitor (Figure 3D and E).
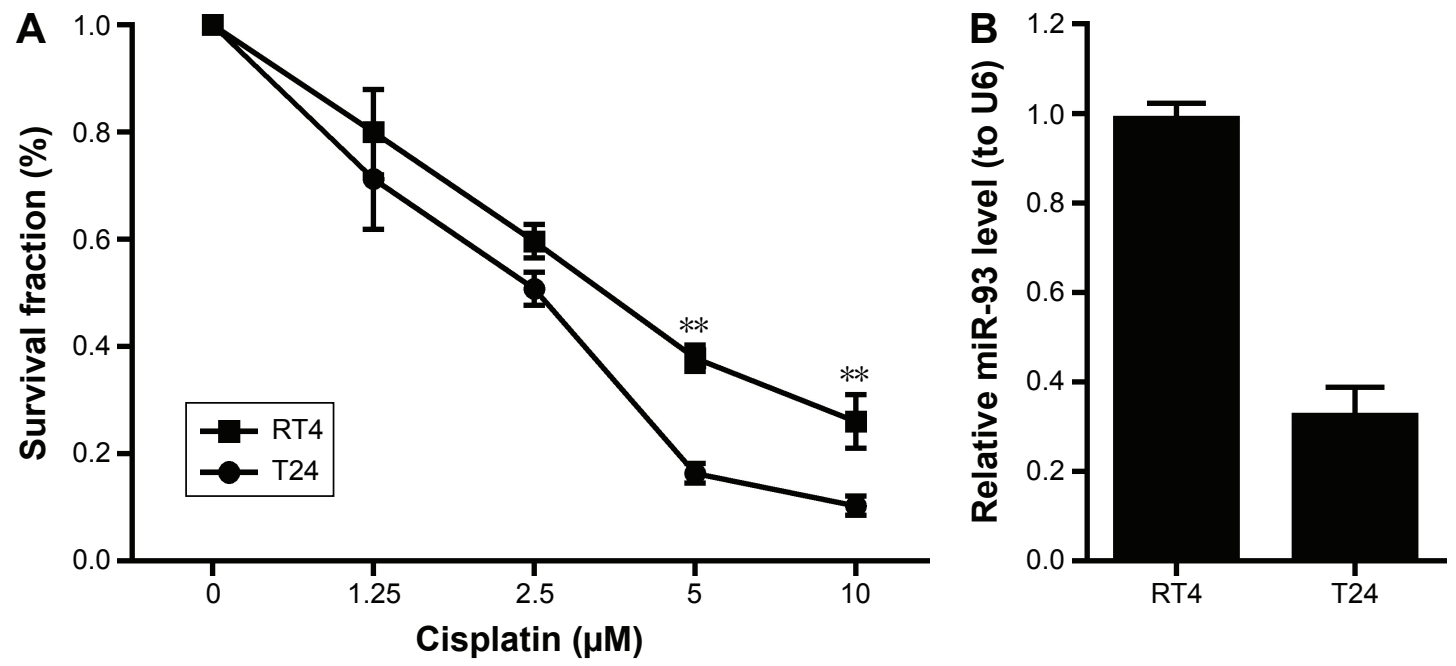

Figure I Expression of miR-93 is decreased in chemo-sensitive bladder cancer specimens and cell lines.

Notes: (A) T24 and RT4 cells $\left(3 \times 10^{5}\right)$ were seeded into six-well plates. The plates were irradiated with cisplatin with doses of 0 , I.25, 2.5, 5, and I0 $\mu$ M given in a single fraction. Colonies containing 50 cells or more were counted. Surviving fraction was the number of colonies/(cells inoculated plating efficiency). $* * P<0.01$. (B) Expression of miR-93 in T24 and RT4 cell lines was examined by quantitative real time PCR. Each bar represents the mean \pm standard deviation of three experiments.

Abbreviation: PCR, polymerase chain reaction. 
A
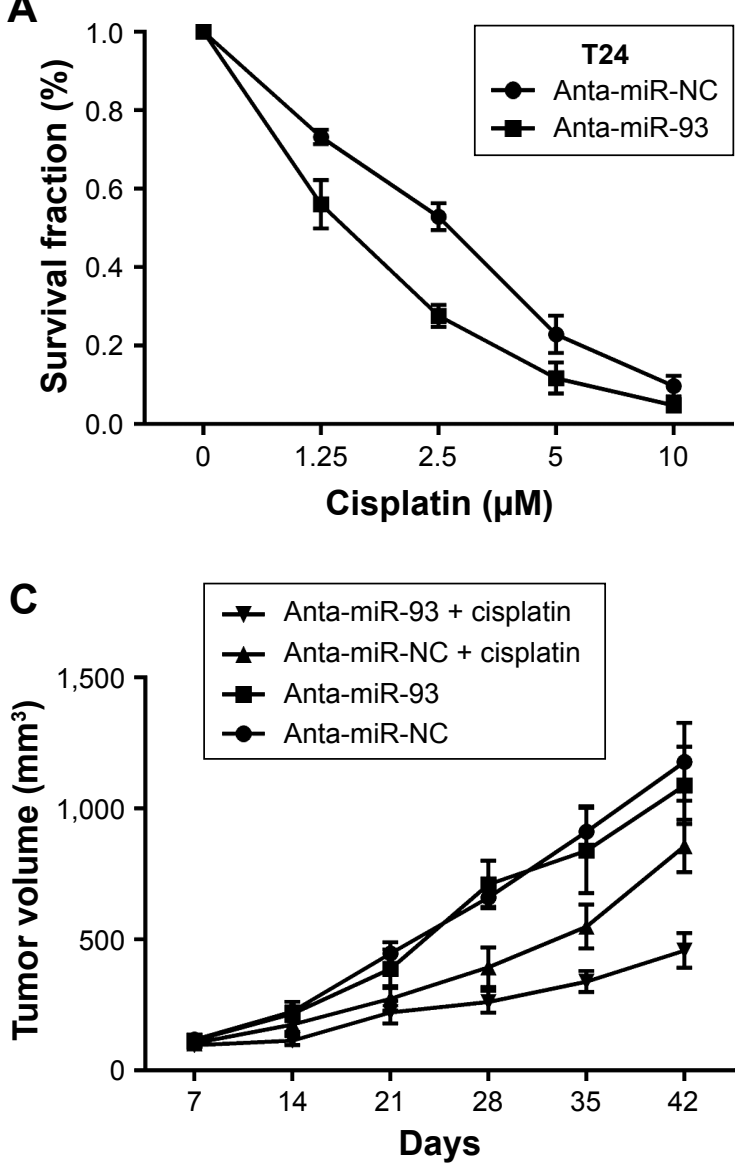

B
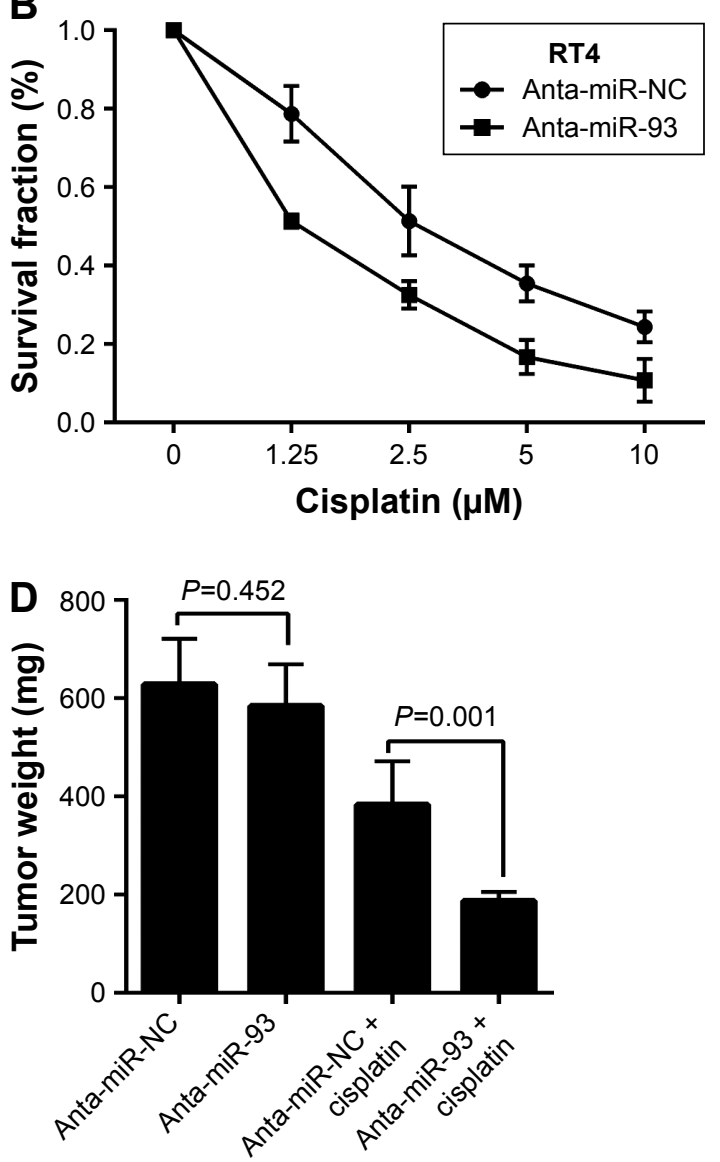

Figure 2 Effects of miR-93 on chemo-sensitivity of bladder cancer cells in vitro and in vivo.

Notes: (A) T24 and (B) RT4 cells $\left(1 \times 10^{5}\right)$ were seeded into six-well plates and subjected to transfection with an miR-93 inhibitor the next day. Twenty-four hours after transfection, the plates were co-cultured with cisplatin with doses of $0,1.25,2.5,5$, and $10 \mu \mathrm{M}$ given in a single fraction. After 8 days, the cells were fixed with $10 \%$ paraformaldehyde and stained with $1 \%$ crystal violet in $70 \%$ ethanol. Cell viability under the treatment of cisplatin was shown as fold change of cell viability normalized to that of the cells treated with saline. Each bar represents the mean \pm standard deviation. The results shown were repeated in three independent experiments. (C) and (D) T24 cells (5×10\%/mouse) stably transfected with miR-93 antagomir (Anta) or control antagomir were injected into the right thigh of 5-week-old BALB/C nude mice. When tumors reached $8 \mathrm{~mm}$ in diameter, the mice bearing either miR-93-repressing tumors or control antagomir tumors were randomly divided into two groups. One group received cisplatin treatment $(5 \mu \mathrm{M})$, and the other group served as controls. Tumor sizes were measured twice a week. There was no difference in tumor weights and volumes between the group transfected with miR-93 antagomir and the group transfected with control antagomir, but after receiving chemotherapy, the tumor weights and volumes of the stably repressing miR-93 group were significantly lower than those of control antagomir group. Each bar represents the mean \pm standard deviation. P-values: Student's t-test.

Abbreviation: NC, negative control.

\section{Functional role of LASS2 in miR-93- modulated chemo-sensitivity}

To further confirm whether the effect of miR-93 on the chemo-sensitivity of $\mathrm{BC}$ cells was dependent on the regulation of LASS2 expression, we first used a luciferase reporter assay to investigate whether miR-93 directly binds to LASS2, leading to its dysregulation. We constructed $\mathrm{pLUC}$ reporter luciferase vectors containing the putative wild-type target site of the LASS2 3'UTR, and transfected them into H293 cells with miR-93 mimics or control mimics. The results showed that miR-93 did not decrease the luciferase activity of the LASS2 3'UTR reporter relative to the negative control group (Figure 4A). Then we employed a gene over-expression approach to examine the effect of miR-93 on LASS2 mRNA and protein expression. RT4 cells were transfected with miR-93 mimics, and LASS2 mRNA and protein expression was measured by quantitative RT-PCR and a Western blot analysis. As shown in Figure 4B and C, over-expression of miR-93 significantly suppressed LASS2 protein expression but not mRNA expression. Next, LASS2 shRNA was transfected into BC cells, and the expression of miR-93 was down-regulated with antagomir. Although the sensitivity to cisplatin treatment was still enhanced, down-regulation of miR-93 only had a very slight effect on cisplatin sensitivity in BC cells when LASS2 was silenced (Figure 4D). These data suggest that the effects of miR-93 on the cisplatin sensitivity of BC cells were achieved, at least in part, by the regulation of LASS2. 


\section{Discussion}

In this study, we identified miR-93 as a critical effector of bladder cancer chemotherapy. Both in vitro and in vivo experiments demonstrated that the suppression of miR-93 could enhance chemo-sensitivity by repressing the expression of LASS2, an anti-oncogene, but not by targeting the 3'UTR of the LASS2 gene.

Many studies of the miRNA profile in bladder cancer have been conducted, including in muscle-invasive $\mathrm{BC},{ }^{20,21}$ low-grade non-invasive $\mathrm{BC},{ }^{22}$ and non-invasive $\mathrm{BC},{ }^{23-26}$ but few studies have investigated the chemo-resistance of BC. Kozinn et al conducted a microarray analysis comparing
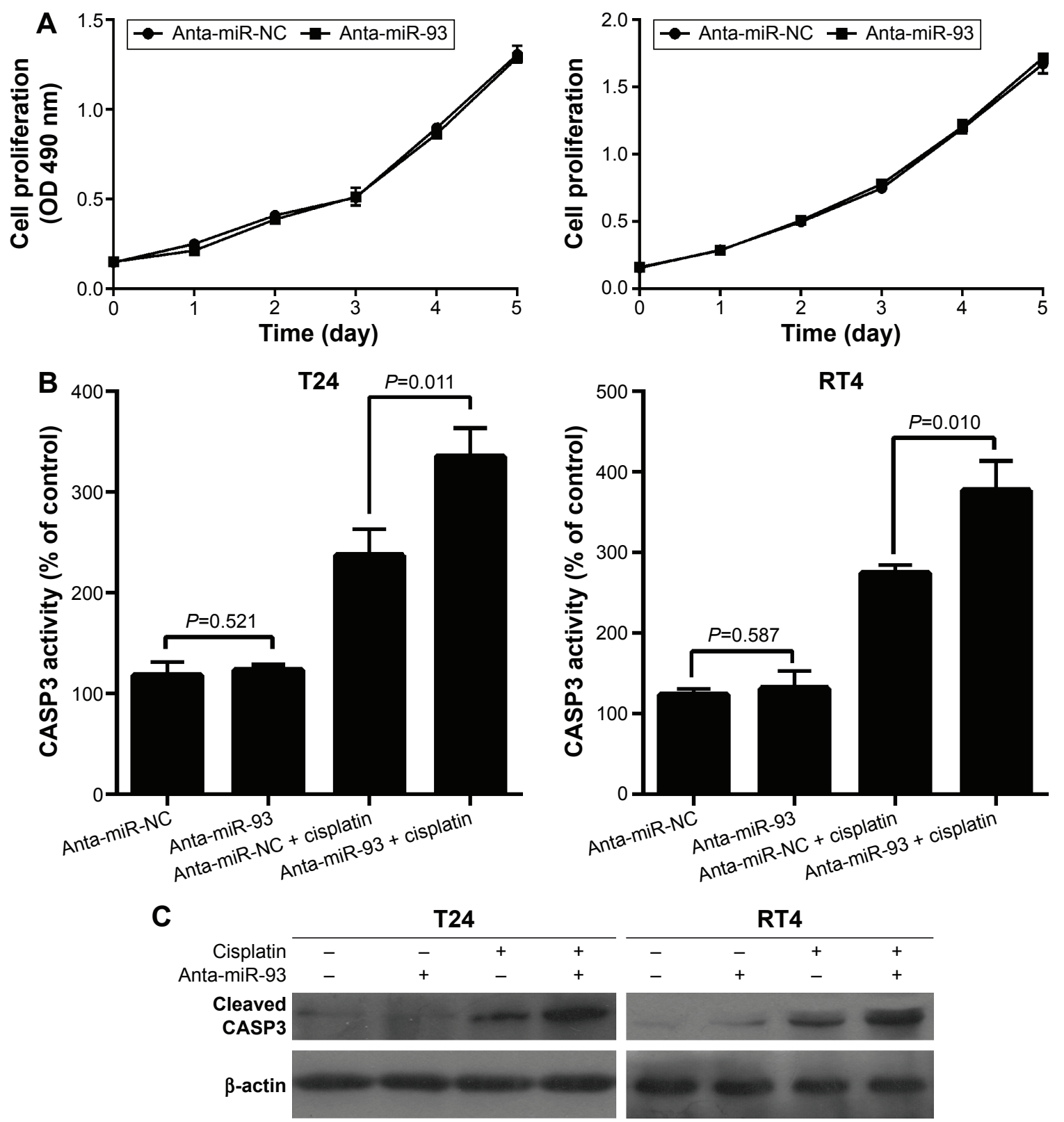

miRNA expression between gemcitabine-resistant and parental cells and identified the differential expression of 66 miRNAs. ${ }^{27}$ Nordentoft et al profiled the expression of 671 miRNAs, and identified 15 miRNAs that correlated with a response to cisplatin-based chemotherapy. ${ }^{18}$ In this study, our miRNA profile results were consistent with the previous studies; some of the same miRNAs were differentially expressed. We tried to combine these miRNA results with our previous research regarding the LASS2 gene. According to results from a target gene prediction, several miRNAs (miR-93, miR-133a, and miR-101) were found to have a nucleotide sequence complementary to the

Figure 3 (Continued) 
D

Control

Cisplatin
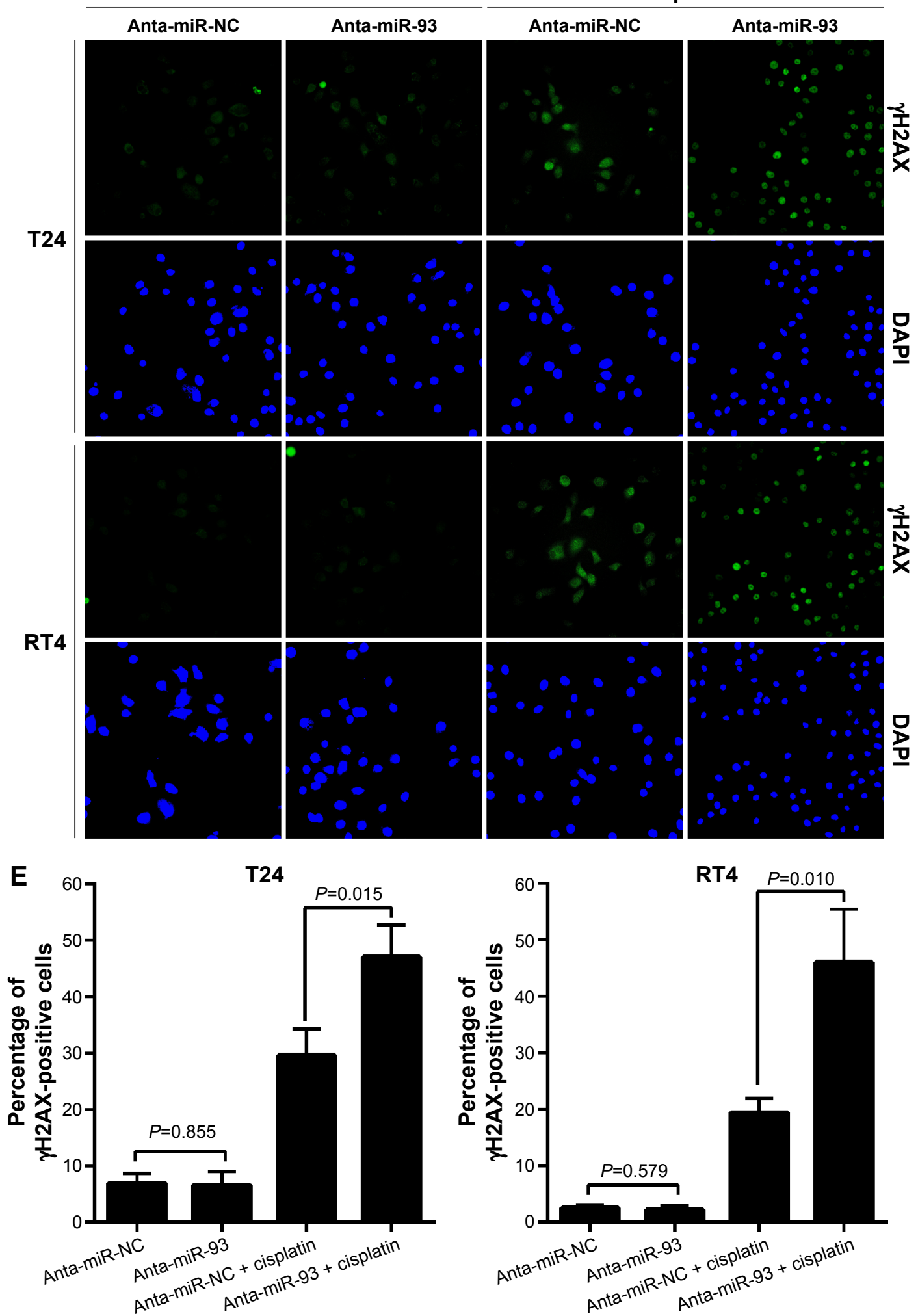

Figure 3 MiR-93 inhibitor enhanced cisplatin-induced cell death by regulation of DNA damage-induced apoptosis.

Notes: (A) miR-93 had no effect on bladder cancer cell growth. (B) Repressing miR-93 did not change the activity of CASP3, but after receiving chemotherapy, activity was detected in T24 and RT4 cells transfected with miR-93 inhibitor. (C) Western blotting was used to determine the expression of cleaved CASP3 in T24 or RT4 cells transfected with miR-93 inhibitor. (D) The T24 and RT4 cells transfected with miR-93 inhibitor for 24 hours followed by exposure to $5 \mu$ M of cisplatin for additional 24 hours, and analyzed by $\gamma \mathrm{H} 2 \mathrm{AX}$ foci staining. (E) Quantification of $\gamma \mathrm{H} 2 \mathrm{AX}$ foci-positive cells of T24 and RT4 cells transfected with miR-93 inhibitor. The cells with $\gamma \mathrm{H} 2 \mathrm{AX}$ foci $>5$ were counted as positive cells and expressed as a percentage. Results are representative of at least three independent experiments and shown as the mean \pm standard deviation. $P$-values: Student's $t$-test. Abbreviations: Anta, antagomir; NC, negative control; DAPI, 4',6-diamidino-2-phenylindole; OD, optical density. 
A

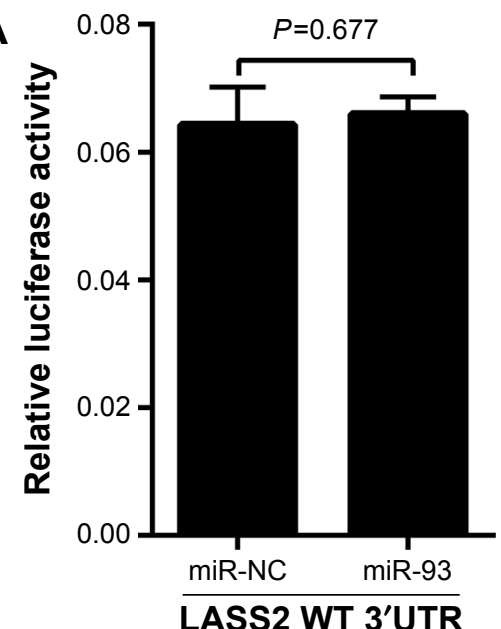

LASS2 WT 3'UTR

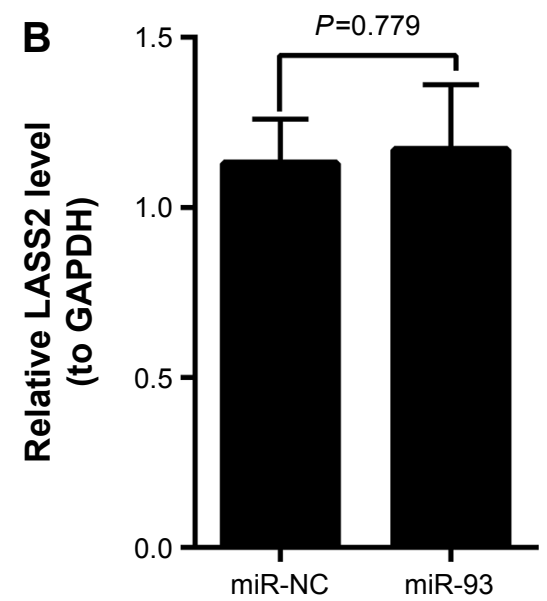

C
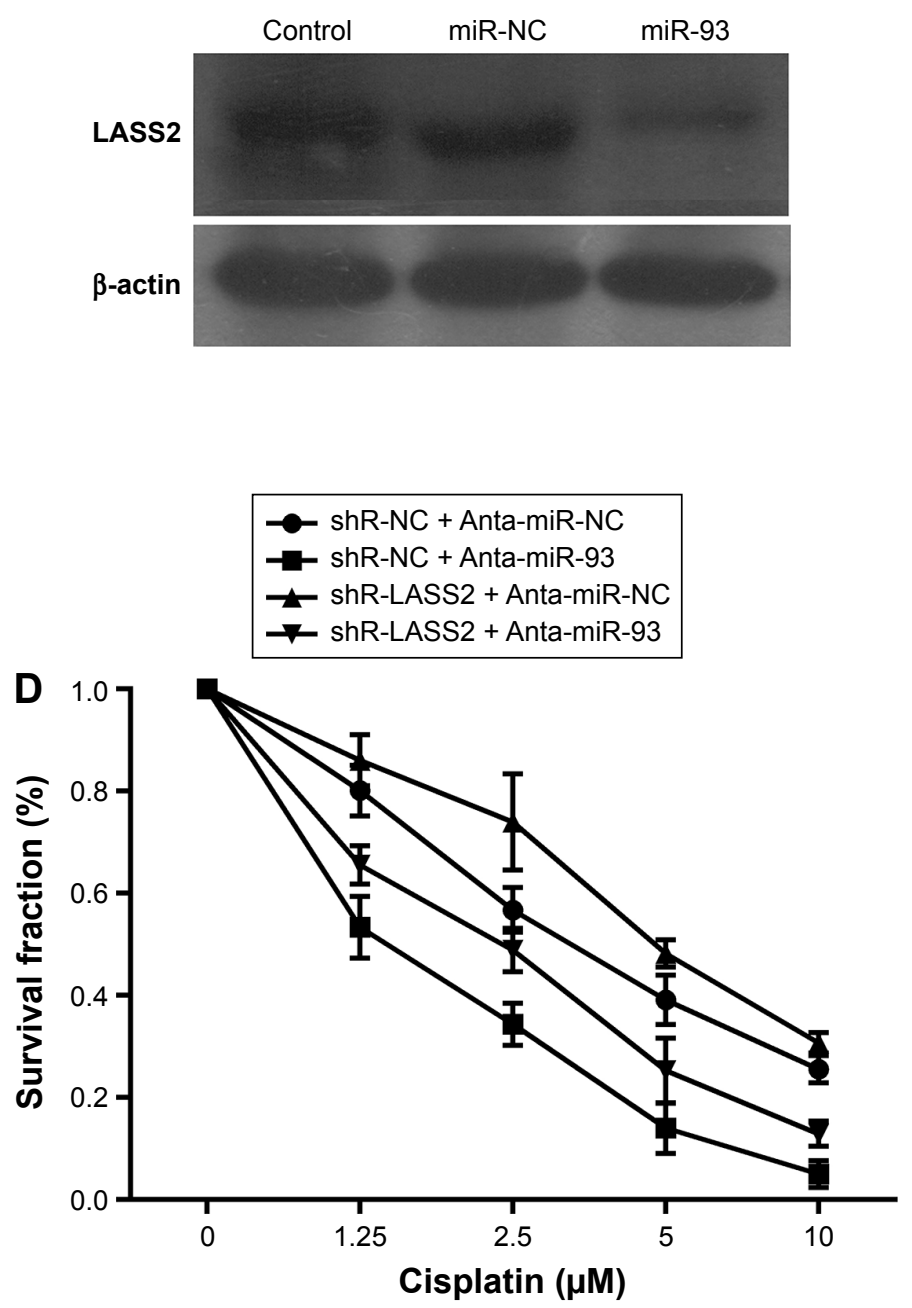

Figure 4 Regulation of LASS2 is involved in miR-93-mediated cisplatin sensitivity.

Notes: (A) The pLUC-WT-LASS2 3'UTR was transfected into 293T cells with miR-NC mimics or miR-93 mimics. Renilla luciferase vector was used as an internal control. RT4 cells were transfected with miRNA mimics. After 48 hours, cells were harvested for real time RT-PCR (B) and Western blot analysis (C). Each bar represents the mean \pm standard deviation. $P$-values: Student's $t$-test. (D) RT4 cells expressing control or LASS2 shRNAs were plated for cisplatin sensitivity assay (mean \pm standard deviation, $\mathrm{n}=3$ ). Abbreviations: UTR, untranslated region; NC, negative control; RT-PCR, reverse transcription-polymerase chain reaction; Anta, antagomir; WT, wild type.

LASS2 3'UTR. MiR-101 was clearly confirmed to affect the chemo-sensitivity of bladder cancer, ${ }^{28}$ while miR-133a was confirmed to inhibit proliferation. ${ }^{29-31}$ Thus, we excluded them and chose miR-93 for further investigation of a probable effect on chemo-sensitivity.

MiR-93 is involved in the progression of several carcinomas. MiR-93 has been reported to act as a promoter or suppressor of various tumors, including prostate cancer, ${ }^{32}$ ovarian cancer, ${ }^{33,34}$ colorectal cancer, ${ }^{35}$ laryngeal squamous cell carcinoma, ${ }^{36}$ and breast cancer. ${ }^{37}$ However, few studies of miR-93 on drug-sensitivity exist. Ohta et al found that miR-93 rendered hepatocellular carcinoma cells more sensitive to sorafenib and tivantinib treatment. ${ }^{38} \mathrm{Fu}$ et al found that miR-93 could regulate chemo-sensitivity to the chemotherapeutic drug cisplatin in ovarian cancer cells by directly targeting PTEN, which participates in the regulation of the AKT signaling pathway. ${ }^{39}$ So far, no study has been found that investigated miR-93 and its effect on chemosensitivity of BC. This study shows, for the first time, that the suppression of miR-93 could enhance chemo-sensitivity of $\mathrm{BC}$ in vitro and in vivo.

Previous studies have shown that silencing LASS2 did not affect cell apoptosis, but inhibited cell apoptosis induced by doxorubicin, suggesting that LASS2 may be related to the chemo-sensitivity of BC cells. MiR-93, a regulator of LASS2, has also been shown to affect the chemo-sensitivity of BC cells. We speculated that LASS2 probably plays an important role in this process. 
LASS2 is an anti-cancer gene that has been associated with many tumors. The aberrant expression of LASS2 has been found in different kinds of cancer, including bladder cancer. ${ }^{40-42}$ The high expression of LASS2 has an inhibitory function in tumor proliferation and metastasis. Our previous study demonstrated that silencing LASS2 promoted the proliferation and metastasis of BC. In this study, the luciferase assay results suggested that LASS2 was not directly targeted by miR-93, but the transfection of miR-93 mimics decreased the protein level of LASS2, but not the mRNA level. Then, to verify that the effect of miR-93 in BC cell chemo-sensitivity was mediated by LASS2, LASS2 was repressed by RNAi, which decreased the BC cell apoptosis induced by cisplatin. Curiously, in our experiments, we could not counter the enhanced effects of miR-93 on BC cell chemo-sensitivity. Down-regulation of miR-93 had a very slight effect on cisplatin sensitivity in BC cells when LASS2 was silenced. We speculated that, because miRNAs can regulate multiple target genes, LASS2 might be only one of them, and other target genes that regulate a LASS2-related signal pathway might have a role in the chemo-sensitivity of bladder cancer, which warrants further investigation.

\section{Conclusion}

In conclusion, we found a causal relationship between the down-regulation of miR-93 and chemo-sensitivity of BC. We also showed that the inhibition of miR-93 could enhance chemo-sensitivity by upregulating the anti-cancer gene LASS2. These findings suggest that miR-93 may be utilized as a new therapeutic target for the treatment of BC.

\section{Acknowledgments}

This work was supported by the National Natural Science Foundation of China (No 81260374, No 81460384), Yunnan Provincial Department of Education Fund (No 2014Z072), Joint Project of Science and Technology, Department of Yunnan and Kunming Medical University (No 2014FA015, No 2014FZ031), Project of Yunnan Provincial Health Department (No 2014NS081), and Project of Yunnan Provincial Science and Technology (No 2015FB196).

\section{Authors' contributions}

All authors contributed toward data acquisition and analysis, took part in either drafting the article or revising it, and agreed to be accountable for all aspects of the work.

\section{Disclosure}

The authors report no conflicts of interest in this work.

\section{References}

1. Raman JD, Jafri SM. Surgical management of bladder urothelial carcinoma with squamous differentiation. Urol Oncol. 2015;33(10):429-433.

2. Kiselyov A, Bunimovich-Mendrazitsky S, Startsev V. Treatment of nonmuscle invasive bladder cancer with Bacillus Calmette-Guerin (BCG): Biological markers and simulation studies. BBA Clin. 2015;4:27-34.

3. Massari F, Santoni M, Ciccarese C, et al. Emerging concepts on drug resistance in bladder cancer: Implications for future strategies. Crit Rev Oncol Hematol. 2015;96(1):81-90.

4. Vallo S, Michaelis M, Rothweiler F, et al. Drug-Resistant Urothelial Cancer Cell Lines Display Diverse Sensitivity Profiles to Potential Second-Line Therapeutics. Transl Oncol. 2015;8(3):210-216.

5. Soukup V, Babjuk M, Bellmunt J, et al. Follow-up after surgical treatment of bladder cancer: a critical analysis of the literature. Eur Urol. 2012;62(2):290-302.

6. Garg M. Prognostic and therapeutic applications of the molecular events in clinical management of urothelial carcinoma of bladder. J Exp Ther Oncol. 2014;10(4):301-316.

7. Balar AV, Milowsky MI. Neoadjuvant therapy in muscle-invasive bladder cancer: a model for rational accelerated drug development. Urol Clin North Am. 2015;42(2):217-224, viii-ix.

8. Eulalio A, Huntzinger E, Izaurralde E. Getting to the root of miRNAmediated gene silencing. Cell. 2008;132(1):9-14.

9. Bernstein E, Caudy AA, Hammond SM, Hannon GJ. Role for a bidentate ribonuclease in the initiation step of RNA interference. Nature. 2001;409(6818):363-366.

10. Sevignani C, Calin GA, Siracusa LD, Croce CM. Mammalian microRNAs: a small world for fine-tuning gene expression. Mamm Genome. 2006; 17(3):189-202.

11. Lewis BP, Burge CB, Bartel DP. Conserved seed pairing, often flanked by adenosines, indicates that thousands of human genes are microRNA targets. Cell. 2005;120(1):15-20.

12. Orellana EA, Kasinski AL. MicroRNAs in Cancer: A Historical Perspective on the Path from Discovery to Therapy. Cancers (Basel). 2015;7(3):1388-1405.

13. Liz J, Esteller M. IncRNAs and microRNAs with a role in cancer development. Biochim Biophys Acta. 2016;1859(1):169-176.

14. Braicu C, Cojocneanu-Petric R, Chira S, et al. Clinical and pathological implications of miRNA in bladder cancer. Int J Nanomedicine. 2015;10:791-800.

15. Noon AP, Catto JW. Noncoding RNA in bladder cancer: a specific focus upon high-risk nonmuscle invasive disease. Curr Opin Urol. 2014;24(5):506-511.

16. Drayton RM, Dudziec E, Peter S, et al. Reduced expression of miRNA-27a modulates cisplatin resistance in bladder cancer by targeting the cystine/glutamate exchanger SLC7A11. Clin Cancer Res. 2014;20(7):1990-2000.

17. Vinall RL, Ripoll AZ, Wang S, Pan CX, deVere White RW. MiR-34a chemosensitizes bladder cancer cells to cisplatin treatment regardless of p53-Rb pathway status. Int J Cancer. 2012;130(11):2526-2538.

18. Nordentoft I, Birkenkamp-Demtroder K, Agerbaek M, et al. miRNAs associated with chemo-sensitivity in cell lines and in advanced bladder cancer. BMC Med Genomics. 2012;5:40.

19. Fan S, Niu Y, Tan N, et al. LASS2 enhances chemosensitivity of breast cancer by counteracting acidic tumor microenvironment through inhibiting activity of V-ATPase proton pump. Oncogene. 2013;32(13): $1682-1690$.

20. XuZ, YuYQ, Ge YZ, et al. MicroRNA expression profiles in muscle-invasive bladder cancer: identification of a four-microRNA signature associated with patient survival. Tumour Biol. 2015;36(10):8159-8166.

21. Pignot G, Cizeron-Clairac G, Vacher S, et al. microRNA expression profile in a large series of bladder tumors: identification of a 3-miRNA signature associated with aggressiveness of muscle-invasive bladder cancer. Int J Cancer. 2013;132(11):2479-2491.

22. Dip N, Reis ST, Abe DK, et al. Micro RNA expression and prognosis in low-grade non-invasive urothelial carcinoma. Int Braz J Urol. 2014;40(5):644-649. 
23. Jin N, Jin X, Gu X, Na W, Zhang M, Zhao R. Screening biomarkers of bladder cancer using combined miRNA and mRNA microarray analysis. Mol Med Rep. 2015;12(2):3170-3176.

24. Song T, Xia W, Shao N, et al. Differential miRNA expression profiles in bladder urothelial carcinomas. Asian Pacific J Cancer Prev. 2010;11(4):905-911.

25. Han Y, Chen J, Zhao X, et al. MicroRNA expression signatures of bladder cancer revealed by deep sequencing. PloS One. 2011;6(3):e18286.

26. Dyrskjot L, Ostenfeld MS, Bramsen JB, et al. Genomic profiling of microRNAs in bladder cancer: miR-129 is associated with poor outcome and promotes cell death in vitro. Cancer Res. 2009;69(11): 4851-4860.

27. Kozinn SI, Harty NJ, Delong JM, et al. MicroRNA Profile to Predict Gemcitabine Resistance in Bladder Carcinoma Cell Lines. Genes Cancer. 2013;4(1-2):61-69.

28. Bu Q, Fang Y, Cao Y, Chen Q, Liu Y. Enforced expression of miR-101 enhances cisplatin sensitivity in human bladder cancer cells by modulating the cyclooxygenase-2 pathway. Mol Med Rep. 2014;10(4):2203-2209.

29. Uchida Y, Chiyomaru T, Enokida H, et al. MiR-133a induces apoptosis through direct regulation of GSTP1 in bladder cancer cell lines. Urol Oncol. 2013;31(1):115-123.

30. Yoshino H, Chiyomaru T, Enokida H, et al. The tumour-suppressive function of miR-1 and miR-133a targeting TAGLN2 in bladder cancer. Br J Cancer. 2011;104(5):808-818.

31. Chiyomaru T, Enokida H, Tatarano S, et al. miR-145 and miR-133a function as tumour suppressors and directly regulate FSCN1 expression in bladder cancer. Br J Cancer. 2010;102(5):883-891.

32. Choi N, Park J, Lee JS, et al. miR-93/miR-106b/miR-375-CICCRABP1: a novel regulatory axis in prostate cancer progression. Oncotarget. 2015;6(27):23533-23547.
33. Chen Q, Qin R, Fang Y, Li H. Berberine Sensitizes Human Ovarian Cancer Cells to Cisplatin Through miR-93/PTEN/Akt Signaling Pathway. Cell Physiol Biochem. 2015;36(3):956-965.

34. Chen X, Chen S, Xiu YL, Sun KX, Zong ZH, Zhao Y. RhoC is a major target of microRNA-93-5P in epithelial ovarian carcinoma tumorigenesis and progression. Mol Cancer. 2015;14:31.

35. Tang Q, Zou Z, Zou C, et al. MicroRNA-93 suppress colorectal cancer development via Wnt/beta-catenin pathway downregulating. Tumour Biol. 2015;36(3):1701-1710.

36. Xiao X, Zhou L, Cao P, Gong H, Zhang Y. MicroRNA-93 regulates cyclin $\mathrm{G} 2$ expression and plays an oncogenic role in laryngeal squamous cell carcinoma. Int J Oncol. 2015;46(1):161-174.

37. $\mathrm{Hu} \mathrm{J}, \mathrm{Xu} \mathrm{J}, \mathrm{Wu} \mathrm{Y}$, et al. Identification of microRNA-93 as a functional dysregulated miRNA in triple-negative breast cancer. Tumour Biol. 2015;36(1):251-258.

38. Ohta K, Hoshino H, Wang J, et al. MicroRNA-93 activates c-Met/PI3K/ Akt pathway activity in hepatocellular carcinoma by directly inhibiting PTEN and CDKN1A. Oncotarget. 2015;6(5):3211-3224.

39. Fu X, Tian J, Zhang L, Chen Y, Hao Q. Involvement of microRNA-93, a new regulator of PTEN/Akt signaling pathway, in regulation of chemotherapeutic drug cisplatin chemosensitivity in ovarian cancer cells. FEBS Lett. 2012;586(9):1279-1286.

40. Wang H, Zhang W, Zuo Y, et al. miR-9 promotes cell proliferation and inhibits apoptosis by targeting LASS2 in bladder cancer. Tumour Biol. 2015;36(12):9631-9640.

41. Zhao Q, Wang H, Yang M, Yang D, Zuo Y, Wang J. Expression of a tumor-associated gene, LASS2, in the human bladder carcinoma cell lines BIU-87, T24, EJ and EJ-M3. Exp Ther Med. 2013;5(3):942-946.

42. Wang H, Wang J, Zuo Y, et al. Expression and prognostic significance of a new tumor metastasis suppressor gene LASS2 in human bladder carcinoma. Med Oncol. 2012;29(3):1921-1927.
OncoTargets and Therapy

\section{Publish your work in this journal}

OncoTargets and Therapy is an international, peer-reviewed, open access journal focusing on the pathological basis of all cancers, potential targets for therapy and treatment protocols employed to improve the management of cancer patients. The journal also focuses on the impact of management programs and new therapeutic agents and protocols on

\section{Dovepress}

patient perspectives such as quality of life, adherence and satisfaction The manuscript management system is completely online and includes a very quick and fair peer-review system, which is all easy to use. Visit http://www.dovepress.com/testimonials.php to read real quotes from published authors. 